\section{Short Technical Report High-Throughput Conformation-Sensitive Gel Electrophoresis for Discovery of SNPs}

\author{
BioTechniques 30:334-340 (February 2001)
}

\begin{abstract}
High-throughput screening for single nucleotide polymorphisms (SNPS) or mutations can be achieved by inexpensive technologies. We modified the original protocols of conformation-sensitive gel electrophoresis (CSGE) to increase throughput several fold to 1.3 samples/min, which is about five times faster than denaturing high-performance liquid chromatography (DHPLC). The modifications include decreasing the gel thickness, increasing the number of lanes to 96, and increasing the number of samples per lane to seven. This high-throughput CSGE method is fast, robust, and as simple as the original protocols. Together with a two-stage strategy for screening homozygotes and the replacement of ethidium bromide with $S Y B R^{\circledR}$ Gold DNA dye staining, this protocol is a reliable and cost-effective alternative for laboratories that require high-throughput screening.
\end{abstract}

\section{INTRODUCTION}

In the post-genomic era, many highthroughput methods are available to screen for single nucleotide polymorphisms (SNPs) or mutations, including automated fluorescent sequencing, denaturing high-performance liquid chromatography (DHPLC) (21), DNA microarrays $(4,10,12)$, single-strand conformation polymorphism-capillary electrophoresis (SSCP-CE) (19), microplate-array diagonal-gel electrophoresis (MADGE) (7) and matrix-assisted laser desorption/ionization time-offlight (MALDI-TOF) (11). Some of these techniques (DNA microarrays, MADGE, and MALDI-TOF) are more suitable for identifying known mutations, while others (automated sequencing, DHPLC, and SSCP-CE) can type both known and novel mutations.

The ideal high-throughput mutationscreening method should possess the following elements. It should be cheap, not requiring expensive equipment with high running and maintenance costs. It should be simple, no more complex than conventional electrophoresis. Finally, it must be fast. The above-mentioned technologies satisfy most of these criteria, but the high price tag is prohibitive to laboratories with limited budgets. However, to achieve highthroughput screening, such state-ofthe-art methods are not indispensable. Some traditional and inexpensive mutation identification technologies, such as conformation-sensitive gel electrophoresis (CSGE), are capable of achieving higher throughput on appropriate modifications of the protocols.

CSGE is a rapid and simple method for the detection of sequence variations in DNA fragments based on differences in the electrophoretic mobility of homoduplexes and heteroduplexes on semi-denaturing polyacrylamide gels $(5,9,14)$. It has been reported to detect more sequence alterations than SSCP analysis (17). Recently, CSGE has been widely used for discovering novel SNPs and mutations in disease genes such as BRCAl $(2,6,17)$ and PTEN (1) in breast cancer, $P L E C 1$ in epidermolysis bullosa simplex with muscular dystrophy (3), TSC1 and TSC2 in tuberous sclerosis (15), and MET in familial renal oncocytoma (22). We use CSGE in our laboratory to identify novel sequence alterations of TIGR/MYOC in primary open angle glaucoma $(16,18)$.

One drawback of CSGE is its inability to detect homozygous sequence alterations because homoduplexes of different SNPs in the same PCR product usually display the same mobility (13). This was tested by Ganguly et al. (9) by generating artificial homoduplexes of DNA fragments identical except for single base changes. Occasionally, some homozygous alterations generated homoduplexes with different mobilities from their wild-type counterparts $(3,21)$. We have also found that homozygous sequence variants usually have the same mobility as their normal strands. Moreover, the traditional staining procedure requires the handling of large volumes of carcinogenic ethidium bromide solution. Here, we describe an improved high-throughput CSGE (HTCSGE) method for safe and reliable screening of both heterozygous and homozygous SNPs or mutations in a large number of DNA samples. By using HTCSGE, we can achieve a throughput of $1.3 \mathrm{samples} / \mathrm{min}$, which is five times faster than DHPLC. HTCSGE is particularly suitable for laboratories that cannot afford the new expensive technologies but still need to achieve high throughput. This new method is simple to perform and is an improvement in speed over both traditional screening methods (such as SSCP) and new methods (such as DHPLC).

\section{MATERIALS AND METHODS}

\section{CSGE and HTCSGE Gel Format, Sample Preparation, and Running}

Model S2001 sequencing gel electrophoresis apparatus (Life Technologies, Rockville, MD, USA) was used. A standard $300 \times 400 \times 0.8 \mathrm{~mm}$ CSGE gel was prepared with $2.5 \mathrm{~mL} 20 \times$ TTE buffer, pH 9.0 (1.8 M Tris base, $4 \mathrm{mM}$ EDTA, 0.6 M taurine), $37.5 \mathrm{~mL} 40 \%$ acrylamide, $15 \mathrm{~mL}$ formamide, $10 \mathrm{~mL}$ ethylene glycol, $33 \mathrm{~mL}$ water, $0.15 \mathrm{~g}$ 1,4-bis(acryloyl)piperazine (PDA crosslinker; Bio-Rad Laboratories, Hercules, CA, USA), $0.1 \%$ ammonium persulfate, and $70 \mu \mathrm{L}$ TEMED. A 0.8-mm 32-well square-tooth comb was used for standard gel preparation. For HTCSGE, the gel format is exactly the same as for standard CSGE, except the gel thickness and the amounts of the ingredients were decreased by half, and two halfwidth double fine sharkstooth combs of $0.4 \mathrm{~mm}$ thickness (Life Technologies) were used to facilitate the loading of 96 samples on one row. The gels were prerun in $0.5 \times$ TTE for $30 \mathrm{~min}$ at $25 \mathrm{~W}$ before sample loading. To prepare heteroduplexes for CSGE loading, $3 \mu \mathrm{L}$ each PCR product was mixed with $3 \mu \mathrm{L}$ loading dye (containing $0.25 \%$ bromophenol blue, $0.25 \%$ xylene cyanol $\mathrm{FF}$, and $30 \%$ glycerol), denatured at $95^{\circ} \mathrm{C}$ for $5 \mathrm{~min}$ and annealed at room temperature for $1 \mathrm{~h}$. For standard CSGE, the $6 \mu \mathrm{L}$ was loaded on a well, while, for HTCSGE, $1 \mu \mathrm{L}$ was loaded. A DNA ladder was loaded in the first 
lane to help track the orientation of the gel after staining. After electrophoresis at $40 \mathrm{~W}$ for $30 \mathrm{~min}$ for HTCSGE and 45 min for CSGE, another batch of sam ples was loaded. This was repeated until the loading was finished. After electrophoresis for $8.5 \mathrm{~h}$ (from the first loading) for seven loadings or $9 \mathrm{~h}$ for eight loadings, the gel was blotted on Whatman ${ }^{\circledR}$ 3-mm paper for staining with $1 \mu \mathrm{L} \mathrm{SYBR}{ }^{\circledR}$ Gold solution (Molecular Probes, Eugene, OR, USA) in 7.5 $\mathrm{mL}$ water. The staining solution was spread evenly over the gel surface for several minutes before exposure to UV light and documentation by a Bio-Rad ${ }^{\circledR}$ Gel-Doc $^{\text {TM }} 2000$ System (Bio-Rad Laboratories).

\section{HTCSGE Experiment}

To test the loading capacity of HTCSGE, a wild-type 386-bp TIGR/MYOC gene exon 1 product (16) with two of its SNP variants, $-83(\mathrm{G} \rightarrow \mathrm{A})$ heterozygote and $47(\mathrm{C} \rightarrow \mathrm{T})$ heterozygote, were amplified by PCR and loaded on the gel in this pattern: $-83(\mathrm{G} \rightarrow \mathrm{A})$ heterozygote, wild-type, $47(\mathrm{C} \rightarrow \mathrm{T})$ heterozygote. The same pattern was repeated 32 times to fill all of the wells. Figure 1 shows seven loadings.

\section{Determination of Detection Limit for Homozygotes by Mixing}

A range of ratios of homozygote to wild-type PCR products was analyzed by CSGE to determine the detection limit. Five sequence alterations were located in four different products of the TIGR/MYOC gene $(16):-2084(\mathrm{~A} \rightarrow \mathrm{G})$ in a 480 -bp product, $-1081(\mathrm{~A} \rightarrow \mathrm{G})$ in a 500-bp product, $-83(\mathrm{G} \rightarrow \mathrm{A})$ in a $386-\mathrm{bp}$ product, and either $136(\mathrm{C} \rightarrow \mathrm{T})$ or 227 $(\mathrm{G} \rightarrow \mathrm{A})$ in a 310 -bp product. PCR products were prepared using $30 \mathrm{ng}$ genomic DNA as template and were then mixed in homozygote:wild-type ratios of $1: 1,1: 2,1: 4,1: 8,1: 16,1: 32$, or $1: 64$ before denaturation and annealing, followed by standard CSGE.

\section{RESULTS AND DISCUSSION}

\section{High-Throughput CSGE}

We used two half-width double fine sharkstooth combs of $0.4 \mathrm{~mm}$ thickness to increase the loading capacity of a CSGE gel. As a result, every row can accommodate 97 samples (96 test samples +1 marker). The thickness of the gel was decreased by half to $0.4 \mathrm{~mm}$. This increases the resolution of the bands and the mutation detection sensitivity of the gel. For DNA fragments of 350-500 bp, we could achieve at least seven loadings per run without such an overlap. This fits in well with usual CSGE practice because CSGE works

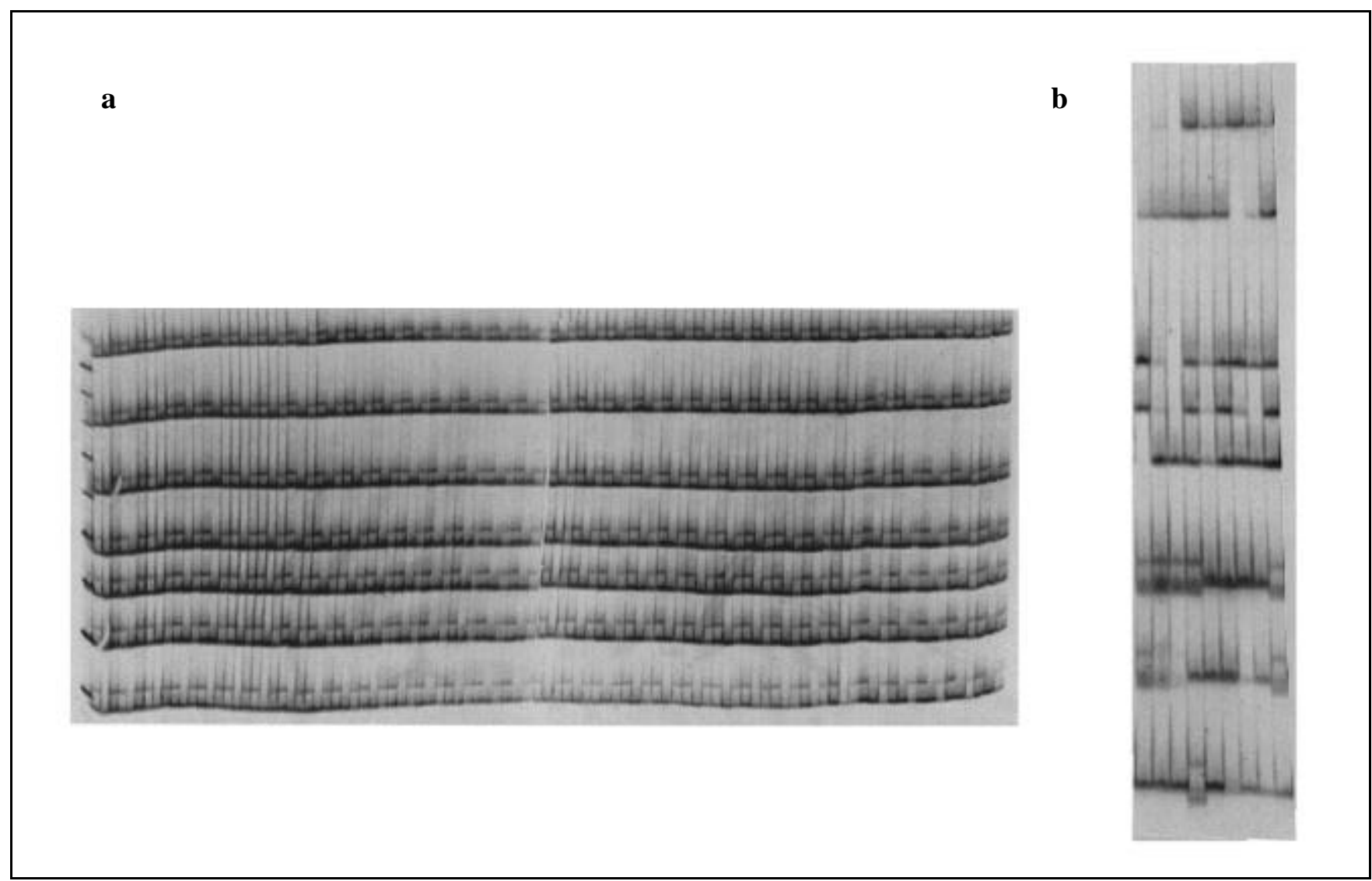

Figure 1. High-throughput CSGE. (a) HTCSGE can resolve 97 samples per row. Seven rows are shown in the figure. The gel was cut into halves to facilitate image capturing. A 50-bp marker was loaded on the left-hand side to assist in orientation. A 386-bp wild-type product and two of its SNP variants, $-83(\mathrm{G} \rightarrow \mathrm{A})$ heterozygote and $47(\mathrm{C} \rightarrow \mathrm{T})$ heterozygote, were loaded repetitively for 32 times in this pattern: $-83(\mathrm{G} \rightarrow \mathrm{A})$ heterozygote, wild-type, $47(\mathrm{C} \rightarrow \mathrm{T})$ heterozygote. (b) HTCSGE can resolve eight rows of samples without overlap of the earliest PCR products with excess oligonucleotide primers from the last loading. 


\section{Genotyping Techniques}

well for DNA fragments around $450 \mathrm{bp}$ in size (14). A HTCSGE gel with seven loadings is shown in Figure 1a, although up to eight loadings could be taken without a decrease in resolution (Figure 1b). Loading more than eight samples in one lane might create an overlap of the first loaded samples with the residual PCR oligonucleotide primers from the last loading.

Therefore, $672(7 \times 96)$ or $768(8 \times$ 96) samples can be analyzed on one gel loaded with seven or eight samples in one lane, respectively. Consequently, a throughput of 1.3 samples/min can be attained for seven loadings per gel and $8.5 \mathrm{~h}$ electrophoresis or 1.4 samples/ min for eight loadings/gel and $9 \mathrm{~h}$ electrophoresis. The throughput of DHPLC was recently reported as 0.25 sam ples/min (8). Therefore, our HTCSGE format is five to six times faster than DHPLC.

HTCSGE is not fully automatic and is relatively labor intensive compared to automated high-throughput technologies. Since it does not require any special equipment other than conventional manual gel apparatus, it offers an alternative method for inexpensive high-throughput screening.

\section{Detection of Both Homozygotes and Heterozygotes by Two-stage CSGE}

Figure 2 shows that CSGE can detect heterozygotes of each of the five sequence alterations as a double band. Most homozygotes run as a single band at the same mobility as wild-types, with the exception of $-1081(\mathrm{~A} \rightarrow \mathrm{G})$ and perhaps $227(\mathrm{G} \rightarrow \mathrm{A})$. As expected, artificial heterozygotes, created by $1: 1$ or 1:2 mixtures of homozygotes and wildtypes, generated patterns of double bands indistinguishable from the natural heterozygotes. However, ratios of 1:4 or greater resulted in gradual diminution of the second band, which was still visible for all five sequence alterations at 1:8. It was discernible in two samples at $1: 16$ but not visible in any sam ple at a ratio of 1:64.

Figure 3 illustrates the steps to detect both heterozygotes and homozygotes by CSGE. Homozygotes may be identified by pooling the homoduplex samples, performing CSGE, and sequencing the members of pools that give heteroduplexes. However, one may decide not to bother screening for homozygotes expected to be more rare than a certain threshold. At HardyWeinberg equilibrium, the frequency of homozygotes will be, for low frequencies, the square of half the frequency of heterozygotes. For example, for a threshold of 1 out of 10000 samples, do not screen if the heterozygote frequency is less than $2 \%$.

Figure 2 also demonstrates that one homozygote can be detected in a pool of up to 8-16 samples. To avoid false negatives, we use pools of five samples. Pooling saves time and money. However, if there are many homozygotes, a large number of samples would have to be sequenced. Therefore, each homoduplex sample should instead be screened individually by mixing with one wild-type sample for CSGE and then sequencing any heteroduplexes.

Table 1 shows the number of CSGE

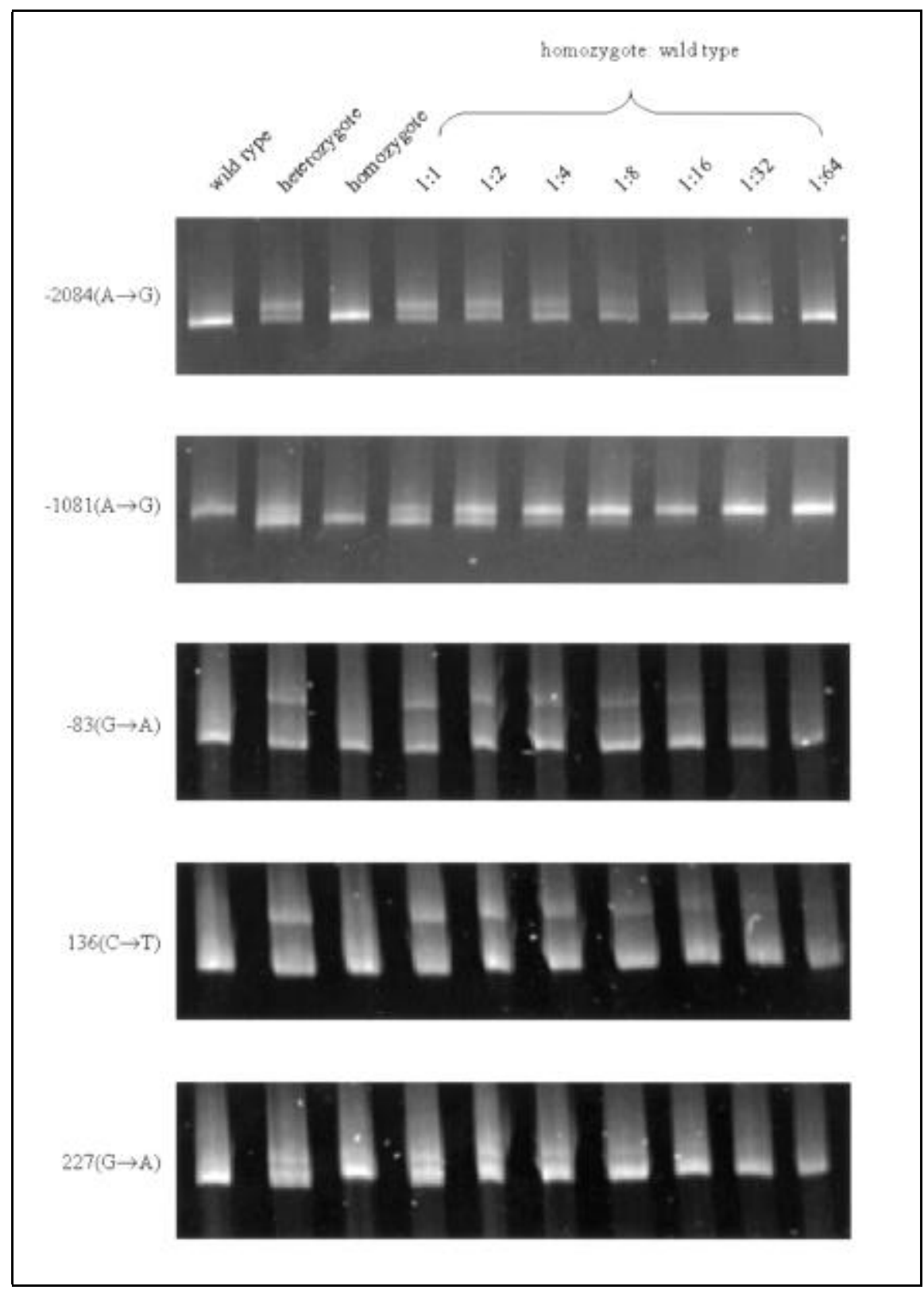

Figure 2. Determination of the CSGE detection limit. The genomic DNAs were quantitated, and $30 \mathrm{ng}$ were used for each PCR. From left to right: wild-type, heterozygote, homozygote, and different dilutions (homozygote:wild-type ratios of 1:1, 1:2, 1:4, 1:8, 1:16, 1:32, and 1:64). 
Table 1. Numbers of Samples that Must Be Analyzed by CSGE or Sequencing when Using the Pooling or Individual Methods to Detect Homozygotes by CSGE

\begin{tabular}{|c|c|c|}
\hline & Pooling & Individual \\
\hline CSGE & $\mathrm{N} / \mathrm{P}$ & $\mathrm{N}$ \\
\hline Sequencing & $\begin{array}{l}\text { MPN (for } \\
\text { small M) }\end{array}$ & $\mathrm{MN}$ \\
\hline \multicolumn{3}{|c|}{$\begin{array}{l}\mathrm{N}=\text { no. of samples, } \mathrm{P}=\text { no. of sam } \\
\text { ples in pool, and } \mathrm{M}=\text { frequency of } \\
\text { homozygotes. }\end{array}$} \\
\hline
\end{tabular}

and sequencing samples with the pooling or individual methods, where $\mathrm{N}=$ the number of samples, $\mathrm{P}=$ the number of samples per pool, and $\mathrm{M}=$ the frequency of homozygotes. Let $S=$ the ratio of the cost (in time and money) of sequencing versus CSGE for one sam ple or pool. The two methods are equally efficient when the CSGE plus sequencing costs of pooling equal the costs of individual screening, or (using the values from Table 1), N/P + SMPN $=\mathrm{N}+\mathrm{SMN}$, or $\mathrm{M}=1 /(\mathrm{SP})$. At HardyWeinberg equilibrium, the frequency of

\section{CSGE each sample}

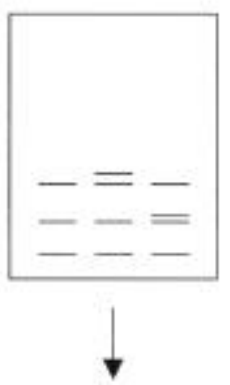

Sequence heteroduplexes

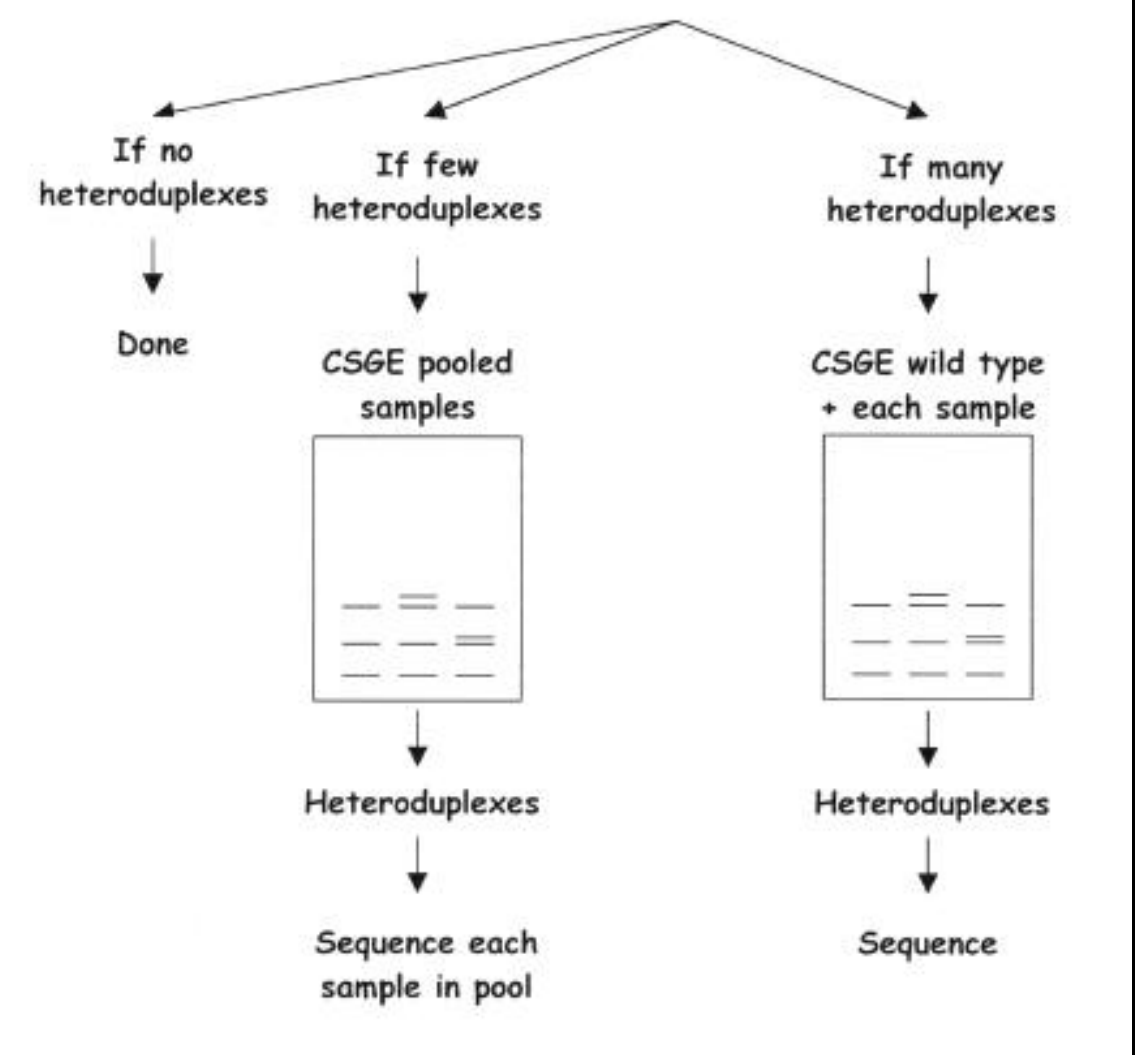

Figure 3. Flowchart for the detection of heterozygotes and homozygotes by CSGE. 
heterozygotes $(T)=2 \sqrt{M}-2 M$. For example, if $\mathrm{P}=5$ and $\mathrm{S}=5$, then $\mathrm{M}=4 \%$ and $\mathrm{T}=32 \%$; thus, it is more efficient to screen individually if $\mathrm{T}$ is greater than $32 \%$. Homozygotes will not be detected in a pool if all of its samples are homozygotes. However, this will only occur with a frequency of $\mathrm{M}^{\mathrm{P}}$, which is insignificant even for the most common polymorphism $(0.1 \%$ for $\mathrm{M}=25 \%$ and $\mathrm{P}=5$ ), unless $\mathrm{P}$ is only 2 or 3 .

This HTCSGE protocol is simple and easy. In short, just screen all the samples once and then screen the normal-appearing samples again (whether pooled or not). In contrast to SSCP, HTCSGE requires only one gel condition at room temperature to detect nearly $100 \%$ of sequence alterations (14), and about 700 samples can be analyzed on one gel.

Ethidium bromide is the most commonly used staining reagent for the detection of DNA bands after gel electrophoresis. It also poses a health hazard to researchers. To improve safety, we used SYBR Gold DNA dye (20), which is structurally similar to SYBR Green and has low mutagenicity. We also reduced the volume of staining solution to $7.5 \mathrm{~mL} / \mathrm{gel}$ to decrease waste and cost. SYBR Gold is also more sensitive than ethidium bromide and is thus more suitable for DNA detection for CSGE.

We conclude that the new HTCSGE protocol is fast, robust, and versatile. It can reliably detect both heterozygotes and homozygotes and eliminates the use of hazardous ethidium bromide. Thus, HTCSGE is suitable for routine mutation screening.

\section{REFERENCES}

1.Carroll, B.T., F.J. Couch, T.R. Rebbeck, and B.L. Weber. 1999. Polymorphisms in PTEN in breast cancer families. J. Med. Genet. 36:94-96.

2.Chang-Claude, J., J. Dong, S. Schmidt, M. Shayeghi, D. Komitowski, H. Becher, M.R. Stratton, and B. Royer-Pokora. 1998. Using gene carrier probability to select high risk families for identifying germline mutations in breast cancer susceptibility genes. J. Med. Genet. 35:116-121.

3.Chavanas, S., L. Pulkkinen, Y. Gache, F.J. Smith, W.H. McLean, J. Uitto, J.P. Ortonne, and G. Meneguzzi. 1996. A homozygous nonsense mutation in the PLEC1 gene in patients with epidermolysis bullosa simplex with muscular dystrophy. J. Clin. Invest. 98:2196-2200.
4.Chee, M., R. Yang, E. Hubbell, A. Berno, X.C. Huang, D. Stern, J. Winkler, D.J. Lockhart, M.S. Morris, and S.P. Fodor. 1996. Accessing genetic information with high-density DNA arrays. Science 274:610-614.

5.Cotton, R.G.H. 1997. Slowly but surely towards better scanning for mutations. Trends Genet. 13:43-46.

6.Couch, F.J., M.L. DeShano, M.A. Blackwood, K. Calzone, J. Stopfer, L. Campeau, A. Ganguly, T. Rebbeck et al. 1997. BRCA1 mutations in women attending clinics that evaluate the risk of breast cancer. N. Engl. J. Med. 336:1490-1415.

7.Day, I.N., E. Spanakis, D. Palamand, G.P. Weavind, and S.D. O'Dell. 1998. Microplate-array diagonal-gel electrophoresis (MADGE) and melt-MADGE: tools for molecular-genetic epidemiology. Trends Biotechnol. 16:287-290.

8.Ezzeldin, H., T. Millender-Swain, S. Jacobson, and S.J. Pittler. 2000. Use of DHPLC for rapid and efficient mutation detection. Invest. Ophthalmol. Vis. Sci. 41:S30.

9.Ganguly, A., M.J. Rock, and D.J. Prockop. 1993. Conformation-sensitive gel electrophoresis for rapid detection of single-base differences in double-stranded PCR products and DNA fragments: evidence for solvent-induced bends in DNA heteroduplexes. Proc. Natl. Acad. Sci. USA 90:10325-10329.

10.Graves, D.J. 1999. Powerful tools for genetic analysis come of age. Trends Biotechnol. 17:127-134

11.Griffin, T.J. and L.M. Smith. 2000. Singlenucleotide polymorphism analysis by MALDI-TOF mass spectrometry. Trends Biotechnol. 18:77-84.

12.Hacia, J.G. and F.S. Collins. 1999. Mutational analysis using oligonucleotide microarrays. J. Med. Genet. 36:730-736.

13.Henderson, B.G., P.R. Wenham, J.P. Ashby, and G. Blundell. 1997. Detecting familial defective apolipoprotein B-100: three molecular scanning methods compared. Clin. Chem. 43:1630.

14.Korkkö, J., S. Annunen, T. Pihlajamaa, D.J. Prockop, and L. Ala-Kokko. 1998. Conformation sensitive gel electrophoresis for simple and accurate detection of mutations: comparison with denaturing gradient gel electrophoresis and nucleotide sequencing. Proc. Natl. Acad. Sci. USA 95:1681-1685.

15.Kwiatkowska, J., J. Wigowska-Sowinska, D. Napierala, R. Slomski, and D.J. Kwiatkowski. 1999. Brief report: mosaicism in tuberous sclerosis as a potential cause of the failure of molecular diagnosis. N. Engl. J. Med. 340:703-707.

16.Lam, D.S.C., Y.F. Leung, J.K.H. Chua, L. Baum, D.S.P. Fan, K.W. Choy, and C.P. Pang. 2000. Truncations in the TIGR Gene in individuals with and without primary open angle glaucoma. Invest. Ophthalmol. Vis. Sci. 41:1386-1391.

17.Markoff, A., H. Sormbroen, N. Bogdanova, S. Preisler-Adams, V. Ganev, B. Dworniczak, and J. Horst. 1998. Comparison of conformation-sensitive gel electrophoresis and single-strand conformation polymorphism analysis for detection of mutations in the BRCA1 gene using optimized conformation analysis protocols. Eur. J. Hum. Genet. 6:145150.

18.Pang, C.P., Y.F. Leung, J.K. Chua, L. Baum, D.S. Fan, and D.S. Lam. 2000. Novel TIGR sequence alteration Val53Ala. Hum. Mutat. (Online) 15:122.

19.Ren, J. 2000. High-throughput single-strand conformation polymorphism analysis by capillary electrophoresis. J. Chromatogr. B. Biomed. Sci. Appl. 741:115-128.

20.Tuma, R.S., M.P. Beaudet, X. Jin, L.J. Jones, C.Y. Cheung, S. Yue, and V.L. Singer. 1999. Characterization of SYBR Gold nucleic acid gel stain: a dye optimized for use with 300-nm ultraviolet transilluminators. Anal. Biochem. 268:278-288.

21.Underhill, P.A., L. Jin, R. Zemans, P.J. Oefner, and L.L. Cavalli-Sforza. 1996. A pre-Columbian Y chromosome-specific transition and its implications for human evolutionary history. Proc. Natl. Acad. Sci. USA 93:196-200.

22. Weirich, G., G. Glenn, K. Junker, M. Merino, S. Storkel, I. Lubensky, P. Choyke, S. Pack et al. 1998. Familial renal oncocytoma: clinicopathological study of 5 families. J. Urol. 160:335-340.

Financial support from the Industrial Support Fund, Hong Kong, is gratefully acknowledged. Address correspondence to Professor C.P. Pang, Department of Ophthalmology \& Visual Sciences, The Chinese University of Hong Kong, Hong Kong Eye Hospital, 3/F, 147K Argyle Street, Kowloon, Hong Kong.e-mail: cppang@cuhk.edu.hk

Received 17 August 2000; accepted 10 October 2000.

Y.F. Leung, P.O-S. Tam, W.C. Tong, L. Baum, K.W. Choy, D.S.C Lam, and C.P. Pang The Chinese University of Hong Kong, Hong Kong 